\title{
New confinement effects on the viscosity of suspensions
}

\author{
P. Peyla ${ }^{1}$ and C. Verdier ${ }^{1}$ \\ 1 CNRS - Université de Grenoble I, UMR5588, Laboratoire Interdisciplinaire de Physique (LIPhy) \\ 38041 Grenoble, France
}

PACS 47.50. Ef - Measurements
PACS 47.57. E- - Suspensions
PACS $47.57 . Q \mathrm{k}$ - Rheological aspects

\begin{abstract}
We present rheological experiments of confined suspensions at moderate concentrations. The analysis is carried out in the framework of a previous study on particle suspensions [Davit and Peyla, EPL, 83, 64001 (2008)] where simulations revealed the presence of unusual effects attributed to confinement, i.e. when the gap size $(h)$ becomes closer to the particle size (d). Deviations from the usual viscosity trends were found. The present work investigates these features further and confirms the important role of the confinement. Extensions are made from the classical approach to the case of confined suspensions where the importance of the reduced gap $\frac{h}{d}$ is taken into account.
\end{abstract}

Introduction. - The viscosity of solid suspensions and pastes is important for industrial applications such as chemical, petrol engineering, food industry as well as in the field of environment [1] where more complex fluids are investigated. The rheology of suspensions is an evolving field where new measurement techniques are of interest $[2,3]$ but where classical rheometrical tools are still proper devices for carrying out accurate measurements.

Even when mastering the rheology of certain fluids, it can become quite difficult to study particular complex flows, such as the ones possessing free surfaces or those generated by complex geometries. An important recent topic is the one of confined flows [4-8] where interfaces or the presence of walls can give rise to intriguing new regimes. This study of confinement has been addressed previously [7] and proposed a possible way to determine viscosity or yield stress parameters using squeezing flows (implying smaller and smaller gaps), assuming that steady states were obtained. But this method raised the question of such steady states in particular since clusters of particles in complex fluids can lead to reorganization of substructures involving longer waiting times for a steady state [9]. Another drawback of the latter method [7] was that a known constitutive equation was needed to derive proper results (in fact a Herschel-Bulkley model). Most studies on confined suspensions deal usually with concentrated systems $[7,8,10]$. Another recent approach of confinement was based on the study of fluctuations and oscillations in concentrated colloidal suspensions leading to shear-thickening [6]. Finally, concentrated emulsions can also behave differently in confined flows [2] and exhibit a characteristic cooperation length which is concentrationdependent.

In a recent study, the authors revealed the presence of anomalous effects due to the confinement of suspensions at moderate concentrations [11]. These effects were attributed to the presence of walls that change the nature of hydrodynamic interactions between the particles. The steady shear viscosity $\eta$ was shown to behave differently from theories for unconfined suspensions. In general, previous authors [12-15] have proved that for moderate concentrations, the viscosity of a non-confined suspension of hard spherical particles behaves as

$$
\eta=\eta_{0}\left(1+a_{1}^{\infty} \phi+a_{2}^{\infty} \phi^{2}\right)
$$

where $\eta_{0}$ is the suspending fluid viscosity, $\phi$ is the volume concentration, and the symbol " $\infty$ " denotes the limit for unbounded domain (i.e. not confined). Eq. (1) is a Taylor expansion where the linear term $\left(a_{1}^{\infty} \phi\right)$ holds for very dilute suspension and $a_{1}^{\infty}=2.5$ has been calculated by Einstein $[12,13]$. The quadratic term $\left(a_{2}^{\infty} \phi^{2}\right)$ represents the contribution of the two-body hydrodynamic interaction. Batchelor and Green [14] gave the first good estimation of $a_{2}^{\infty}=5.2 \pm 0.3$ for homogeneous suspensions, this term being calculated more precisely later [15] and found close to $a_{2}^{\infty}=5$. But the relationship (1) seems to fail as suspensions are confined, so that the usual con- 
vex evolution predicted by eq. (1) from Batchelor et al. $\left(a_{2}^{\infty}>0\right)$ changes to become concave. This means that the coefficient $a_{2}^{\infty}$ becomes negative while confining the suspension. This is due to wall effects which change completely the hydrodynamic interactions at the microscale. Macrorheology can reveal this microscopic behavior by measuring the effective viscosity as a function of $\phi$ for confined suspensions. One may argue that continuum mechanics theories only hold when the volume to be studied is much larger than the volume occupied by a typical particle in the sample (with diameter $d$ ), therefore that the viscosity should not depend on such effects. Still it is important for practical applications (e.g. flow of confined suspensions through holes or tubes, like in ball bearings) to get an idea about the relationship between the so-called effective viscosity (measured in a rheometer) and gap size.

In this study, an attempt is made to correlate experimental results with the numerical ones obtained prevously [11] at moderate concentrations. Rheometrical studies are carried out in order to obtain typical low-shear viscosity data, as the gap $(h)$ is reduced, for a large number of concentrations up to $20 \%$. The results are analyzed along these lines and these results are pushed further in order to come up with practical relationships in terms of the concentration $\phi$ and the ratio $\frac{h}{d}$, which measures confinement.

In the first part, rheometrical techniques are presented, as well as care which has been taken in order to obtain such data. Then a discussion proposes to analyze the results in terms of reduced parameters. Simple relationships are obtained and the main conclusions are summarized.

Rheometrical measurements. - Rheometrical measurements were carried out on a stress-controlled rheometer (Bohlin, Gemini 150, Malvern) at $T=23^{\circ} \mathrm{C}$. A plate-plate geometry was used in order to change the gap easily. Suspensions were prepared by slowly mixing the fluids with particles. The fluids and particles were selected because of their closely matched densities $\left(\rho_{p}=1.05 \mathrm{~g} / \mathrm{cm}^{3}\right.$ for the polystyrene particles and $\rho_{f}=0.978 \mathrm{~g} / \mathrm{cm}^{3}$ for the fluid). The fluid was a Rhodorsil silicone (PDMS 47V60000, Rhodia, viscosity roughly around 60 Pa.s) and was chosen for its Newtonian behavior at the shear rates tested (typically $10 s^{-1}$ ). Its relaxation time is $\lambda=1 \mathrm{~ms}$ and is small enough so that non-Newtonian effects do not affect the viscosity as shown previously $[16,17]$. A viscous PDMS is also a good candidate because larger viscosities slow down sedimentation, so that these effects can be neglected here. Spherical polystyrene particles (Dynoseeds TS80, TS140 and TS230, Danemark) were chosen for their densities, and also because of their monodispersity (see fig. 1). Their diameters were $80 \mu \mathrm{m}, 140 \mu \mathrm{m}$ and $230 \mu \mathrm{m}$ respectively, in order to be able to set final gaps in the range of the particle diameters, i.e. $[100-300 \mu \mathrm{m}]$. A small gap correction was used to avoid tolerance errors, machine precision and concentricity errors, a procedure already used before [18-20]. This led to the accurate determination of the PDMS vis- cosity $\eta_{0}=60.6 \pm 0.9$ Pa.s, in agreement with our previous work [17].

For measuring the suspensions viscosity, rough solid surfaces were available (roughness $5 \mu \mathrm{m}$ ) to avoid slip. Note that it is necessary to use a small roughness since confinement effects are to be studied. Some experiments were repeated twice and showed similar trends as the gap was varied. This is sufficient to assert the no-slip hypothesis. Furthermore, previous works reporting slip in suspensions showed that such effects occur at low shear rates smaller than $0.1 s^{-1}$ typically [1] whereas our materials are tested at a higher shear rate of $10 s^{-1}$. Finally, slip is usually observed at higher concentrations, not below $20 \%$ as in this study.

Suspensions were prepared and degased; then they were set onto the rheometer. The upper plate was gently brought down until contact. Experiments started with large gaps (usually $1500 \mu \mathrm{m}$ ) to obtain the asymptotic value of the viscosity, as postulated from continuum mechanics (the gap in the case of a $230 \mu \mathrm{m}$ diameter particle is then around seven times higher which is good enough to obtain this limit, as will be shown). Then the gap was reduced to smaller values. Excess fluid was removed after each gap change. Several fresh samples were used to cover the gap range. Thus, sedimentation was evaluated to be negligeable. A shear rate of $10 s^{-1}$ was used (see below). Note that due to the presence of particles, small gap experiments usually took longer, of the order of a few minutes. This is due to the internal reorganization of particles under shear that may occur due to the possible formation of particle clusters that are broken or coalesce until equilibrium is obtained [9].

Next a series of experiments were carried out at a constant shear rate of $10 \mathrm{~s}^{-1}$, using different particle sizes and particle concentrations (typically between $0 \%$ and 20\%). This shear rate was used for various complementary reasons. It is a shear rate allowing steady state to be obtained rapidly (few minutes), it is also constant as was used previously in simulations [11], and finally this value ensures that the suspensions remain in the Newtonian regime, and are not shear-thinning. This constant value can be achieved thanks to a feedback loop control available with this rheometer. Note that, in order to match the simulations, low Reynolds numbers $R_{e}=\frac{\rho R^{2} \dot{\gamma}}{\eta_{0}}$ should be used, which is the case here since $R_{e}$ is of the order $10^{-6}$ in the experiments.

The reduced viscosity $\frac{\eta}{\eta_{0}}-1$ was investigated first. All curves are shown on the same graph to start with and one can notice an important dispersion. Indeed fig. 2 shows the raw data at several concentrations $(5-7.5-10-12.5-$ $15-17.5-20 \%)$ and for three typical particle diameters $(80 \mu \mathrm{m}, 140 \mu \mathrm{m}$ and $230 \mu \mathrm{m})$. Note that in some cases, a few data points seem to be more dispersed at small $h / d$ or high concentrations, which does not seem to be satisfactory. These data points were shown to be attributed to the difficulties inherent to the small gap measurements. 
Table 1: Values of parameters $m_{1}, m_{2}, m_{3}$ used as a model fit for the data in fig. 3 .

\begin{tabular}{|c|c|c|c|}
\hline Concentration & $m_{1}$ & $m_{2}$ & $m_{3}$ \\
\hline $2.5 \%$ & 0.058 & 0.58 & 0.88 \\
\hline $5 \%$ & 0.13 & 0.73 & 0.65 \\
\hline $7.5 \%$ & 0.21 & 1.0 & 0.65 \\
\hline $10 \%$ & 0.29 & 1.29 & 0.55 \\
\hline $12.5 \%$ & 0.42 & 1.40 & 0.54 \\
\hline $15 \%$ & 0.55 & 1.60 & 0.50 \\
\hline $17.5 \%$ & 0.70 & 1.50 & 0.40 \\
\hline $20 \%$ & 0.88 & 1.55 & 0.39 \\
\hline
\end{tabular}

As mentioned before, for small gaps, plate parallelism is important as well as tools concentricity effects or the fact that some particles may be in contact with the wall or touch one another. This leads to reduced accuracy as the viscosity rises rapidly, especially for high concentrations. These data points were kept in the next figures and only concern a few measurements for each concentration, particularly when $h<1.5 d$.

It is then proposed to explain this data in terms of the reduced gap $h / d$, as shown in fig. 3. Data points superpose quite well together for all particle diameters. Note that the limiting values of the viscosities are obtained for large $h / d$, usually when $h / d>10$ roughly. This whole set of data is the main set of results which is used throughout the rest of the paper.

A typical trend is obtained showing a plateau for the reduced viscosity at large reduced gaps $h / d$, then as $h / d$ becomes smaller, the reduced viscosity $\frac{\eta}{\eta_{0}}-1$ increases similarly for all concentrations. It was found that a simple exponential function of the type $\frac{\eta}{\eta_{0}}-1=m_{1}+m_{2} e^{-m_{3} h / d}$ fits the data quite well. This leads to the following set of parameters in table. 1 ; in particular $m_{1}$ represents the value of $\frac{\eta}{\eta_{0}}-1$ for large reduced gaps $h / d$.

Together with this limit for large $h / d$, other values of $\frac{\eta}{\eta_{0}}-1$ at various values of $h / d$ can now be plotted as a function of $\phi$.

Discussion. - In order to see the relevance of these results in view of previous studies, it is now suggested to look at the dependence of the reduced viscosity in terms of $\phi$ as a truncated series, while keeping only the first two terms in $\phi$, as in eq. (2).

$$
\frac{\eta}{\eta_{0}}-1=a_{1}(h / d) \phi+a_{2}(h / d) \phi^{2}
$$

The exponential fit used in fig. 3 worked very well. It was required for replotting the data for all values of $h / d$ with a very good accuracy (see fig. 4). On the other hand, eq. (2) is more likely to be compared to previous works on suspensions $[11,14,15]$. As it is shown in fig. 4 , the asymptotic expansion should only be valid up to medium range concentration as in the case of large $h / d$, where Cichoki's correction is shown to work only when $0<\phi \lesssim 0.12$. As a comparison, the Krieger-Dougherty model [21], $\left(\frac{\eta}{\eta_{0}}\right)_{K D}=\left(1-\phi / \phi_{m}\right)^{-2.5 \phi_{m}}$, works well for all concentrations in the range studied. In any case, it is clear that a curvature inversion is observed for confined suspensions as can be seen in fig. 4, especially when $h / d<2$. Then we used eq. (2) to predict the values of the coefficients $a_{1}(h / d)$ and $a_{2}(h / d)$, functions of the reduced gap $h / d$, when $\phi \lesssim 0.12$. Good correlations are obtained within measurement uncertainty.

In fig. 5 , values of such parameters $a_{1}(h / d)$ and $a_{2}(h / d)$ used in the asymptotic expansion are shown as a function of confinement. Several comments can be made. First it is demonstrated that as gap size decreases, $a_{1}$ increases, therefore moves away from the constant Einstein's value of 2.5. The latter value is obtained only for large values of $h / d$. The behavior of the second coefficient $a_{2}$ is more complex and is important because it contains the hydrodynamic interactions between particle pairs. This term $a_{2}$ starts with values close to 5 , as predicted earlier [14, 15], and decreases very sharply as confinement increases. This result is similar to the previous numerical study [11] and to a recent work using a semi-analytical treatment [22]. Thus it can be concluded that hydrodynamic interactions in the presence of walls decrease the second term in the expansion until it becomes very small and possibly negative.

The discussion of these results can be related to several previous works. Confinement is believed to affect the hydrodynamic interactions between particles [23]. Goyon et al. [2] showed that cooperative effects can take place through the introduction of a correlation length in the flow of confined concentrated emulsions whereas Frydel et al. [24] found velocity correlations over long distances and long times. Thus our results provide another justification of the viscosity change due to the presence of walls in the vicinity of the particles. Note that the effective viscosity was also measured in capillary tubes (through pressure determination) in the case of concentrated suspensions of red blood cells by Pries et al. [25] and that changes were also observed for high confinements. But this study is the first one to analyze rheometrical data in the context of the well-known Batchelor's eq. (1) for semi-dilute suspensions, which is largely affected by confinement. First, the parameters of the classical formulas $[14,15]$ are not valid any longer as one moves away from the continuum mechanics limit, in our case this limit is met when $7<h / d<10$; then unusual effects appear with higher confinements, as already suggested previously $[11,26]$. Finally simple formulas can be proposed to investigate the dependence of the effective viscosity of the type $\frac{\eta}{\eta_{0}}-1=a_{1}(h / d) \phi+a_{2}(h / d) \phi^{2}$, where $a_{1}(h / d)$ and $a_{2}(h / d)$ have been measured experimentally, and correspond to simulations [11]. Note that we remain in the range of small concentrations such as $\phi \lesssim 0.12$ in order to look for a reduced series expansion in terms of $\phi$. It 
is possible that larger concentrations may be used from this work up to $\phi \sim 0.2$, but then other relations may be needed by modification of the empirical fits by Krieger and Dougherty [21]. Note that for higher concentrations, it may also be necessary to consider the more complex rheology of concentrated suspensions and use other models based on the shear-rate dependence of the viscosity, like shear-dependent [8] or yield-stress fluids [7]. Such fractal models or Herschel-Bulkley laws exist and seem to provide satisfactory results for unconfined suspensions [9,27] but fail in confined concentrated systems [2]. Indeed a cooperative length scale defines the transition $[2,28]$, but it is very small already below $40 \%$. Thus this study precisely falls in the range where no controlled-length phenomena exist. Still it leads to unusual effects. Finally, other effects like ordering transitions may also appear at higher concentrations [10] leading to a different rheology. But this was not the major purpose of this study, for already surprising results were found to arise only by studying the zero-shear viscosity of confined suspensions.

Conclusions. - In this analysis, rheometrical data has been obtained for suspensions as the gap becomes close to the diameter of suspended particles, for moderate concentrations. The results found lead to an extension of Batchelor's work [14] in terms of the reduced gap parameter $h / d$, and simple formulas were derived from experiments. The parameters in eq. (2) were determined so that the relationship between the effective reduced viscosity $\frac{\eta}{\eta_{0}}-1$ and $\phi$ showed a concavity inversion at small reduced gaps $h / d$. This model allows one to apply viscosity corrections when confinement effects become important. It may be realistic to use it in the case of special processes, such as moulding, forming or extrusion of materials which involve suspensions in geometries whose sizes become comparable to particle diameters.

Acknowledgments. - We are grateful to Dr. H. Galliard (Laboratoire de Rhéologie, Grenoble) for providing the PDMS fluid and helpful advice regarding the experiments.

\section{REFERENCES}

[1] Coussot P., Rheometry of pastes, suspensions and granular materials (Wiley-Interscience) 2005.

[2] Goyon J., Colin A., Ovarlez G., Ajdari A. and BocQUeT L., Nature, 454 (2008) 84.

[3] Ovarlez G., Rodts S., Chateau X. and Coussot P., Rheol. Acta , 48 (2009) 831.

[4] Pesché R. and Ngele G., Phys. Rev. E , 62 (2000) 5432.

[5] Bhattacharya S., Blawzdziewicz J. and Wajnryb E., J. Fluid Mech., 541 (2005) 263.

[6] Isa L., Besseling R., Morozov A. N. and Poon W. C. K., Phys. Rev. Letters, 102 (2009) 058302.

[7] Yan Y., Zhang Z., Cheneler D., Stokes J. R. and Adams M. J., Rheol. Acta, 49 (2010) 255.
[8] Brown E., Zhang H., Forman N. A., Maynor B. W., Betts D. E., DeSimone J. M. and Jaeger H. M., J. Rheol. , 54 (2010) 1023.

[9] Quemada D., Eur. Phys. J. AP , 1 (1998) 119.

[10] Yeo K. and Maxey M. R., Phy. Rev. E , 81 (2010) 051502.

[11] Davit Y. and Peyla P., Europhys. Letters , 83 (2008) 64001.

[12] Einstein A., Annals der Physik, 19 (1906) 289.

[13] Einstein A., Annals der Physik, 34 (1911) 591.

[14] Batchelor G. K. and Green J. T., J. Fluid Mech. , 56 (1972) 401.

[15] Cichoki B. and Felderhof B. U., J. Chem. Phys. , 89 (1988) 1049.

[16] Elkissi N., Piau J. M., Attané P. and Turrel G., Rheol. Acta, 32 (1993) 293.

[17] Verdier C. and Brizard M., Rheol. Acta , 41 (2002) 514.

[18] Binding D. M. and Walters K., J. Non-Newtonian Fluid Mech. , 1 (1976) 277.

[19] Connelly R. W. and Greener J., J. Rheol. , 29 (1985) 209.

[20] Kramer J., Uhl J. T. and Prud'homme R. K., Polym. Eng. Sci., 27 (1987) 598.

[21] Krieger I. M. and Dougherty T. J., Trans. Soc. Rheol. I, II (1959) 137.

[22] Sangani A., Acrivos A. and Peyla P., submitted, (2011) .

[23] Diamant H., J. Phys. Soc. Jpn., 78 (2009) 041002.

[24] Frydel D. and Diamant H., Phy. Rev. Letters , 104 (2010) 248302.

[25] Pries A. R., Neuhaus D. and Gaehtgens P., Am. J. Physiol. Heart Circ. Physiol., 263 (1992) 1770.

[26] Cui B., Diamant H., Lin B. and Rice S. A., Phys. Rev. Letters, 92 (2004) 258301.

[27] Iordan A., Duperray A. and Verdier C., Phys. Rev. E, 77 (2008) 011911.

[28] Bonnoit C., Lanuza J., Lindner A. and Clement E., Phys. Rev. Letters, 105 (2010) 108302. 


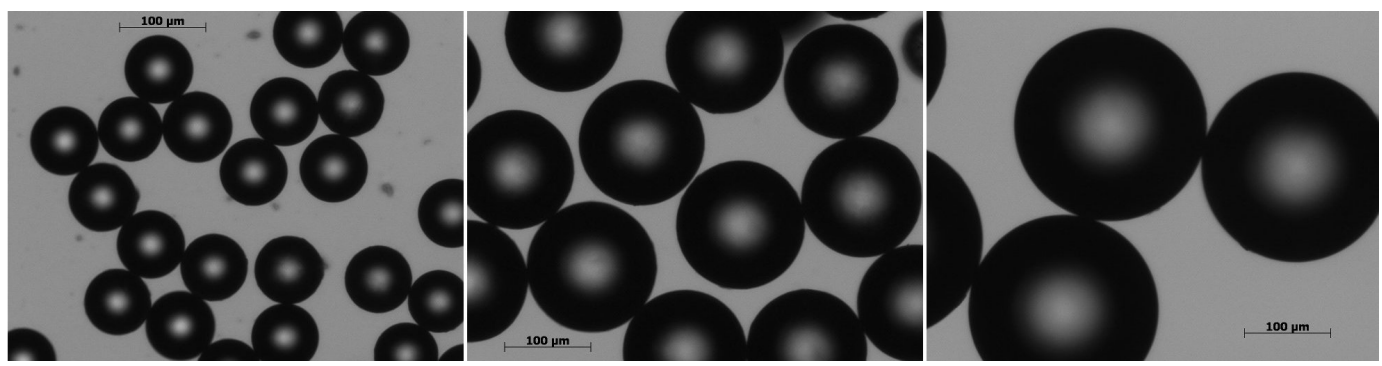

Fig. 1: Microscopy images of the spherical monodisperse polystyrene particles of respective diameters $80 \mu m, 140 \mu m, 230 \mu m$, from left to right. Scale bar is $100 \mu m$ on all three images.

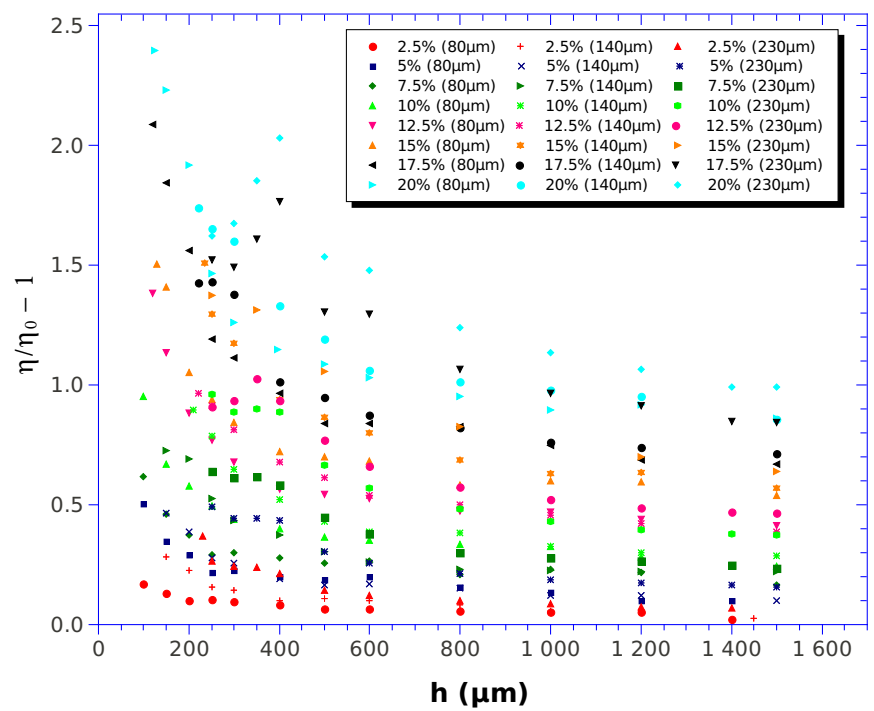

Fig. 2: Reduced viscosity $\frac{\eta}{\eta_{0}}$ vs. gap $h$, at different concentrations $\phi$, and for different particle diameters.

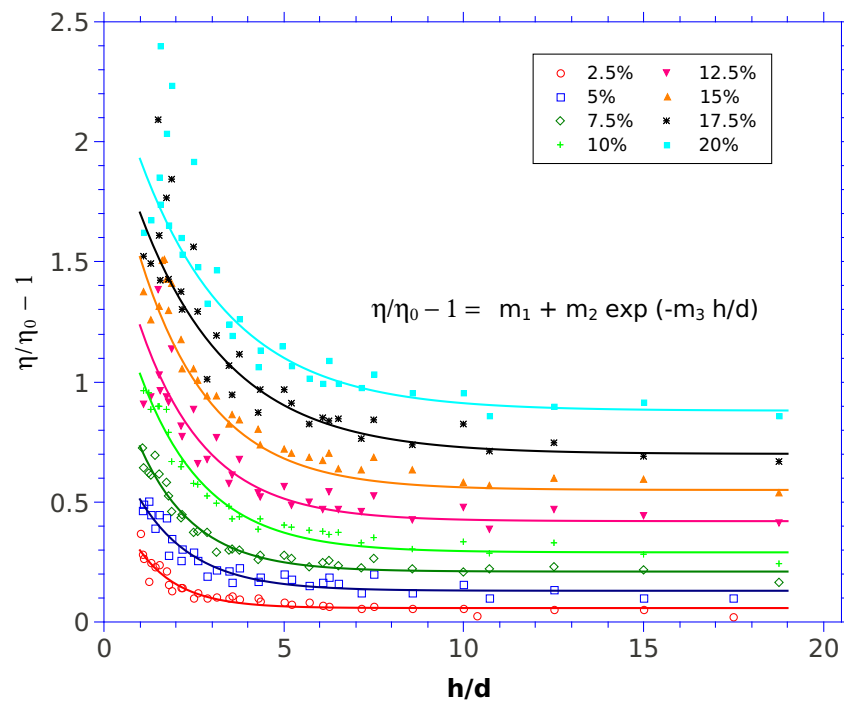

Fig. 3: Master curves showing $\frac{\eta}{\eta_{0}}-1$ vs. $h / d$ at different concentrations and corresponding to particle diameters $80 \mu m, 140 \mu m, 230 \mu m$. Fits of the type $\frac{\eta}{\eta_{0}}-1=m_{1}+m_{2} e^{-m_{3} h / d}$ are also shown, the values of parameters $m_{1}, m_{2}$, and $m_{3}$ can be found in table. 1 . 


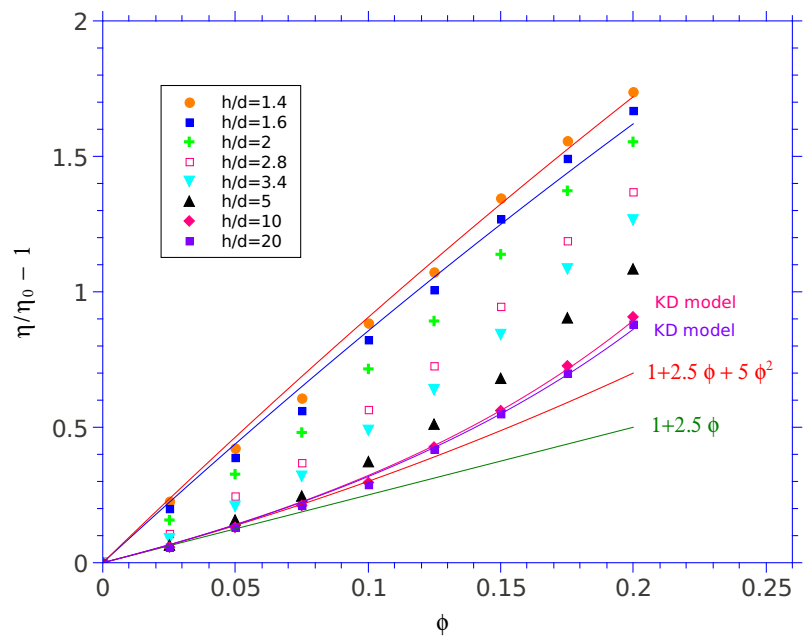

Fig. 4: Values of the reduced viscosity $\frac{\eta}{\eta_{0}}-1$ vs. $\phi$ at different reduced gaps $h / d$. Fits using Krieger-Dougherty $\left(\frac{\eta}{\eta_{0}}\right)_{K D}=$ $\left(1-\phi / \phi_{m}\right)^{-2.5 \phi_{m}}$ with $\phi_{m}=0.5$ and 0.55 are shown to work well at any concentration for high values of $h / d$ (respectively for $h / d=10$ and 20). The classical Einstein law $[12,13] \frac{\eta}{\eta_{0}}=1+2.5 \phi$ and the correction $[14,15] \frac{\eta}{\eta_{0}}=1+2.5 \phi+5 \phi^{2}$ are also shown for comparison. The latter one works well for $0<\phi \lesssim 0.12$. Lines to guide the eye for lower gaps $(h / d=1.4$ and $h / d=1.6)$ show the curvature inversion.

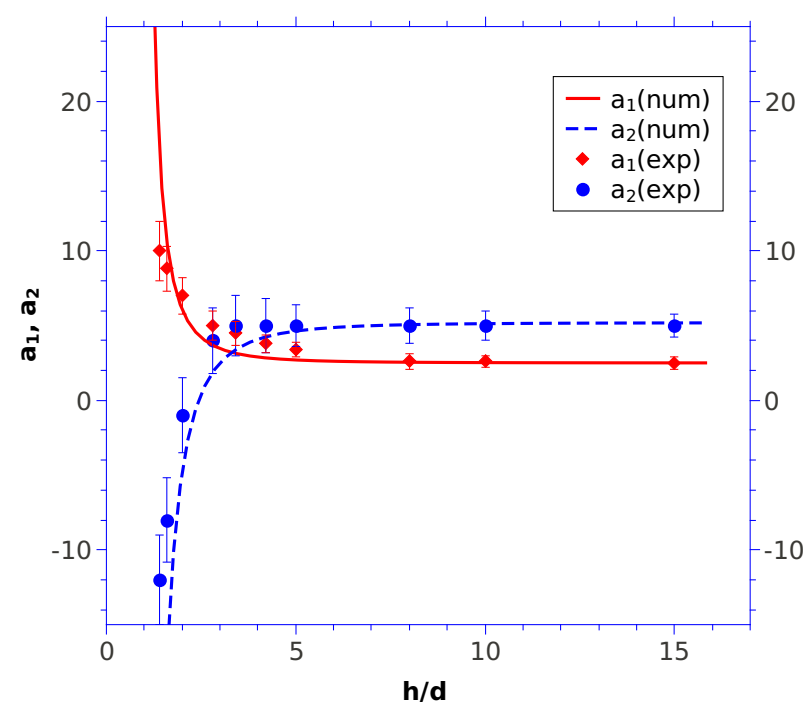

Fig. 5: Values of the parameters $a_{1}$ and $a_{2}$ used in the asymptotic expansion of eq. (2), in terms of the confinement $h / d$. Parameters $a_{1}$ and $a_{2}$ have been adjusted from fig. 4 for $\phi \lesssim 0.12$. The lines correspond to the numerical simulations of Davit et al. [11]. 